\title{
Du bon et du mauvais sel
}

\section{Eberhard Wolff}

PD Dr rer. soc., membre de la rédaction Histoire de la médecine

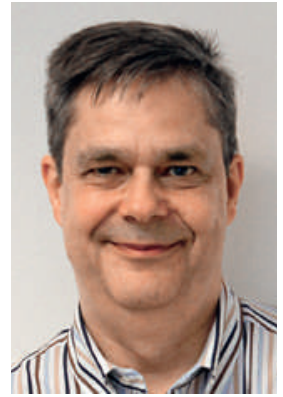

«Au fait, tu pourrais acheter du sel, s'il te plaît?» Cette phrase banale mène tout droit à un nouveau champ de bataille de la vie courante. Même à l'épicerie du coin, il y a le choix entre un "produit naturel» du Jura et une «source de vie» vaudoise, évidemment pure, ainsi que des sels iodés/fluorés ou délibérément sans additifs.

Dès la taille de magasin au-dessus, le problème de la provenance s'amplifie, prenant l'allure de la classique dispute sur la destination de vacances: «Montagne ou mer?» Et le dilemme ne s'arrête pas là. Montagnes suisses ou carrément Himalaya? Sel marin de l'Atlantique ou de la Méditerranée? Et pour aller plus loin: sel marin ordinaire ou fleur de sel récoltée à la main, non raffinée, riche en minéraux et en algues et plutôt dispendieuse? Dans un supermarché au-delà de la frontière, j'ai récemment compté cinquante sels différents en rayon, dont un noir d'Hawaï.

Dans cette vogue actuelle, les sels et produits dérivés sont tout à fait bénéfiques. Même l'un de nos opérateurs téléphoniques s'en est aperçu. Le sel n'est plus seulement un produit gastronomique, il est bienfaisant pour tout le corps, notamment lorsqu'il se pare d'arguments de médecine douce: "Associé à des ingrédients frais, peut être source de nutriments sains», lit-on sur un moulin de sel de l'Atlantique. Sans parler des nombreux soins bien-être externes au sel.

Ce n'est pas vraiment nouveau. Il y a 200 ans environ, les médecins adeptes de bains et de sources vantaient les vertus thérapeutiques du sel pris oralement avec l'eau de cure ou absorbé par la peau lors d'un bain dans une eau salée. Mais même à l'époque, on trouvait des détracteurs. Les pharmaciens considéraient le sel de table comme un simple aliment, éventuellement un laxatif. Les deux groupes échangeaient des arguments académiques, tirant chacun un peu la couverture à soi, selon une étude sur l'histoire du "cristal chatoyant» [1]. Le sel n'a été «diabolisé» qu'à partir de la fin du XIX siècle, d'abord par le mouvement de naturopathie amateur de légumes. A partir des années 20 , le régime pauvre en sel, voire sans sel, du médecin allemand Max Gerson, qui a émigré par la suite, était censé guérir la migraine, puis la tuberculose et enfin le cancer. Le chirurgien Ferdinand Sauerbruch lui fit une forte publicité pour l'époque. En Suisse aussi, des patients se voyaient infliger une nourriture fade sans grand effet [2]. Des études de diverses provenances, reposant sur des conceptions variées, estiment aujourd'hui encore que le sel pose un problème médical.

Depuis les années 20, l'ajout d'iode au sel de table a été encouragé en Suisse, afin de prévenir les affections dues aux carences correspondantes, comme le goitre. Depuis le milieu des années 50, le sel est aussi enrichi en fluor pour éviter les caries. Ces deux additifs ont d'ailleurs fait du sel de cuisine le contraire d'un problème jusqu'à aujourd'hui: un produit de prévention et de santé.

\section{Le sel n'a été «diabolisé» qu'à partir de la fin} du XIX ${ }^{\mathrm{e}}$ siècle.

Depuis les années 70, à peu près, il est rendu responsable de l'hypertension, une théorie vivement contestée, même maintenant. A mots prudents, une initiative de la Fondation Suisse de Cardiologie promeut une baisse de la consommation moyenne de sel, y compris dans cette revue. Là, le sel est encore autre chose: un facteur de risque.

Le champ de sel est le théâtre d'une bataille pour la suprématie des régimes de connaissances et des interprétations. Et ce genre de lutte est rarement totalement flegmatique, dénué d'émotions et d'intérêts. Le sel ressemble en cela à d'autres produits de base comme la graisse [3] ou le sucre, traditionnellement réprouvé. Dernière nouvelle: le 26 novembre sort That Sugar Film, un amusant documentaire tourné en Australie, qui pointe du doigt les sucres cachés. Qu'est-ce qui est le plus grave, l'abus de graisse ou de sucre, demandait Arte en février. Réponse: la combinaison des deux. Après les guides de Tissot, la macrobiotique selon Hufeland et la "réforme de la vie», nous sommes à nouveau dans une phase obsessionnelle d'observation et de régulation de notre mode de vie. Peu importe que le chlorure de sodium soit enrichi en naturel, en pureté, en bien-être, en prévention ou en risque - la prochaine farce de saucisse apparaîtra à coup sûr.

\section{Références}

1 Vogel J. Ein schillerndes Kristall. Eine Wissensgeschichte des Salzes zwischen Früher Neuzeit und Moderne. Cologne, entre autres: Böhlau; 2008.

2 Ritzmann I. Hausordnung und Liegekur. Vom Volkssanatorium zur Spezialklinik: 100 Jahre Zürcher Höhenklinik Wald. Zurich: Chronos; 1998.

3 Colombani P. Fette Irrtümer. Ernährungsmythen entlarvt. Zurich: Orell Füssli; 2010. 